\title{
THE DEVELOPMENT OF PSYCHOPATHOLOGY AS A BRANCH OF SCIENCE.
}

\author{
BY BERNARD HART, LoNDON.
}

THE aim of this paper is to describe, in summary fashion, the history of psychopathology as a branch of science, and to consider how far it has succeeded in establishing its claim to an assured position within the fold of science.

The extent and boundaries of the path we desire to traverse will be made clearer if some preliminary words are devoted to the precise meaning of the terms in which the subject of inquiry has been defined. Psychopathology is to be understood, not as a mere description of mental symptoms, but as an endeavour to explain disorder or certain disorders in terms of psychological processes. Its difference from a mere description of mental symptoms is of the same order as that which exists between clinical medicine and that explanation of the phenomena of clinical medicine in terms of causal processes which constitutes pathology. 'Explain' is used here in the sense in which it constitutes the goal of the method of science. Science is not a compilation of facts, but a method of dealing with our experience. It consists in (1) the recording and classification of phenomenal experience, (2) the finding of formulæ which will serve to resume that experience. This latter part involves the construction of concepts or 'laws,' which will embrace the phenomena we have observed, and enable us to predict the occurrence of further phenomena, the validity of the 'law' being tested by its capacity to fulfil these two conditions. The function of the scientific law and its relationship to the phenomena with which it is concerned may be exemplified by chemical phenomena and the atomic theory, physical phenomena and the law of gravitation, the phenomena of light and heat and the æther theory. It should be observed that these laws are not found or observed by the investigator; they are constructed by him to explain what he has found or observed. The aim of science is to understand and control our phenomenal experience, and the validity of the concepts it constructs is determined by the extent to which they satisfy this aim. Each branch of science claims the right to construct its own concepts, provided that they are constructed according to the rules of scientific method.

That portion of our experience which is constituted by the behaviour 
of living organisms has been attacked by several branches of science, each regarding the phenomena from its own standpoint, and interpreting them in terms of its own concepts. Biology, for example, interprets the phenomena of living organisms in terms of life-process and biological laws, physiology in terms of nervous energy, reflex action, and so forth, chemistry in terms of the interaction of chemical compounds. Some of the phenomena are capable of explanation by the concepts of more than one branch of science, some can be more intelligibly and usefully explained by the concepts of one branch than by those of another, some are at present capable of explanation by the concepts of one branch only. The hope is always before us that the concepts of one branch may ultimately be reduced to the concepts of another, especially when the latter are concepts of a wider validity. There is a reasonable hope, for example, that the concepts of nervous energy and reflex action may ultimately be reduced to the wider concepts of chemistry and physics. But, to a large extent, such a reduction is a goal of the future, and, for the present, each branch must be content to explain whatever phenomena it can in terms of its own concepts, having always in view the essential aim of all science-the understanding and control of our experience by the fashioning of scientifically constructed ' laws.'

Can psychology claim a place as one of the branches of science capable of usefully explaining the phenomena of living organisms ? For a long time this claim was denied, and psychology was treated as an alien with no right of entry into the fold of science, because it dealt with non-material and non-spatial objects, which the crude philosophy of the nineteenth century scientist regarded as necessarily incapable of scientific treatment, and even as 'epiphenomenal' and unreal. So soon as it was realized, however, that science is not defined by the nature of the objects with which it deals, but by the method of investigation applied to those objects, and that its field comprises the whole field of our experience, then the right of psychology to contribute its quota to the explanation of the phenomena presented by living organisms could no longer be gainsaid. Moreover, psychology could claim the right to interpret the phenomena in psychological terms, and to construct psychological concepts in order to explain those phenomena. The only condition, but one rigidly to be observed, was that the concepts must be constructed according to the method of science, that is to say, they must be based on carefully observed experience, they must serve to resume that experience, and they must be verifiable by an appeal to experience.

How far psychology has attempted to carry out this task in the elucidation of certain disorders of the human organism, how far it has succeeded, and what limitations have been found to beset its path, these are the problems which form the subject of this paper. 
There are certain disorders in which the clinical phenomena have a dominantly psychological character, and are only capable of being adequately described in psychological terms. These are the psychoses, comprising the various types of insanity. This sphere would seem to be the most obvious one to attack by a psychological method, and it might have been thought that psychopathology would have found here its most suitable material, and its best chance of successful results. Actually, however, the historical development of psychopathology has taken a different road. The first great advances were made in a field where the most prominent phenomena were not mental at all, the field of hysteria, with its anæsthesias, paralyses, and other disturbances of an apparently physical kind. Physiology had previously attempted to explain hysteria by its conception of 'functional nervous disorder,' but this conception failed to satisfy the canons by which every scientific conception must stand or fall. It was not based on observed experience, but merely on a theoretical assumption designed to bring hysteria into line with organic diseases. It did not enable the investigator to understand the phenomena with which he had to deal, it did not enable him to predict their course and occurrence nor to control their course and occurrence, and it could not be verified by any appeal to experience. It was, in fact, useless, in the sense that a scientific conception, being a weapon with which we hope to achieve an end, is useless if it does not help us towards that end. The way was clear, therefore, for a fresh attempt to explain hysteria, and the foundation of a psychopathological conception was laid by Charcot, when he proposed the view that certain hysterical phenomena were due to 'ideas.' The avenue thus opened was explored by one of Charcot's pupils, Pierre Janet. He investigated the various phenomena of hysteria, and found that they were capable of being interpreted in precise psychological terms, and, finally, he succeeded in formulating a conception which served to explain, in part at any rate, the nature of those phenomena. This conception will be best understood by describing the steps of Janet's researches with regard to one group of hysterical phenomena, functional anæsthesia, and we shall do this in some detail, because it provides an excellent example of the employment by psychopathology of a method which conforms strictly to the method of science. In the first place, it was found that the anæsthesias, although they did not correspond in their distribution to the distribution of any section of the nervous system, did have a distribution which corresponded to something. The familiar glove anæsthesias, for example, ending in sharp lines at the level of the wrist, had a distribution inexplicable by any lesion of the nervous system, but their distribution corresponded precisely to the patient's idea of his own hand. That is to say, the incidence of the symptom was plainly determined by a factor of a psychological order, and it would, therefore, be profitable to 
seek for a psychological conception in order to explain it. Secondly, these anæsthesias exhibited a curious paradoxical character. Patients suffering from extensive anæsthesias involving a whole limb or half the body rarely appeared to sustain any accidental injury to the anæsthetic part, whereas in patients with relatively far smaller organic anæsthesias, syringomyelics for example, such injuries frequently occurred. It would seem, indeed, that the hysterical patient must be able to feel with his anæsthetic limb in order to evade the accidents which would otherwise inevitably befall it. Similarly, patients with hysterical amblyopia of such a degree that the field of vision was reduced to a single point were able to play at ball, a performance obviously impossible unless the greater part of the retina were capable of receiving visual impressions. This paradoxical character was, perhaps, exemplified most clearly by the case of a boy who, after being in a fire, developed hysterical phenomena consisting on the one hand in the occurrence of hysterical fits whenever the patient saw a flame, and on the other hand in an amblyopia whereby the visual field was restricted to 30 degrees. If the boy were tested with a perimeter he was unable to see the paper disc until it had travelled along the perimeter arm to the 30-degree radius. If, however, a lighted match were substituted for the disc of paper, then immediately it reached the limits of normal vision a fit occurred. Quite clearly, therefore, the patient was able to see over the whole field of normal vision, and equally clearly, he was blind to everything outside 30 degrees.

The conception which Janet constructed to explain these phenomena was the conception of 'dissociation of consciousness.' He presumed that consciousness, instead of pursuing its course as a single homogeneous stream, was capable of being split into two or more independent currents, so that the consciousness belonging to one current would be unaware of, and unable to control, that belonging to another contemporaneous current. Hysterical anæsthesia was then explicable as the result of such a dissociation, the sensations from the anæsthetic area not being non-existent, but diverted into a current separated from the main stream of consciousness. Although thus cut off and, therefore, incapable of being perceived by the main stream, they could influence the motor apparatus, and thereby produce just those phenomena which had been observed, the avoidance of injury by the hemianæsthetic and the fits in the blind boy. The conception of dissociation, therefore, served to explain the observed phenomena, and it could, moreover, be experimentally verified. The patient could be hypnotised, for example, and access being thereby obtained to the dissociated portion, the actual existence of the sensations belonging to the anæsthetic area could be conclusively established.

Functional paralyses could be similarly explained, and the conception of dissociation was found to be-applicable to a wide range of 
hysterical phenomena, including amnesias, somnambulisms, and double personality. Janet's work was confirmed and amplified by a number of subsequent investigators, in particular by the extensive and important researches of Dr. Morton Prince, and the value of dissociation as an explanatory concept has now been established beyond question. Certain difficulties appear in applying it to some of the phenomena with which we have to deal, but these are due rather to misapprehension of the nature of the concept than to defects in the concept itself. For example, in many cases of hypnotic somnabulism the hypnotic consciousness is aware of the whole range of the patient's experience, whereas the personal consciousness has no knowledge of the experience belonging to the hypnotic consciousness. This one-sided and non-reciprocal lack of awareness may seem difficult to explain by dissociation, which would appear necessarily to involve a break between the two streams of consciousness equally untraversable in whichever direction it might be attempted, whereas in the example we have cited the break is impassable when viewed from the side of the personal consciousness, and traversable with ease when viewed from the side of the hypnotic consciousness. The difficulty is, however, dependent upon a misconception of the nature of dissociation and an abuse of the spatial metaphor in which it has been defined. Dissociation, of course, does not imply an actual separation in space, and from the nature of the phenomena with which it is concerned it obviously can have no real spatial significance whatever. The dissociation is a functional dissociation, an 'out-of-gear' relationship, and if this is understood, the existence of a non-reciprocal dissociation ceases to be inexplicable. The spatial metaphor, in which psychological concepts are often expressed, is valid and useful so long as its real nature is carefully kept in mind, but it leads easily to abuse and untrustworthy deductions.*

Dissociation may be regarded as the first fruit of psychopathology. It was a conception built up by a strictly scientific method, it illuminated a vast field of phenomena which had hitherto baffled every attempt at explanation, and it opened up the way to therapeutic possibilities in which that control of phenomenal experience which is the ultimate goal of science was abundantly satisfied. Dissociation, however, only takes us a certain distance in the understanding of the phenomena with which we are dealing, and a further step is clearly required to answer the question "Why does dissociation take place?" This further step was attempted by Freud, but before considering the immensely important concepts which he has introduced, it will be desirable briefly to trace out a path of development in psychopathology parallel to that traversed by Janet.

* This danger, for example, has particularlv to be kept in mind in estimating the value of the Freudian psychology, with its extensive use of a complicated spatial terminology in the conceptions of the conscious, pre-conscious and unconscious. 
Psychopathology had approached the problem of hysteria with the aid of another conception, that of 'suggestion.' This conception had had a long historical development, including in its course the observation of certain phenomena by Mesmer, ascribed by him to 'animal magnetism,' the observation and induction of similar phenomena by the hypnotists, and the ascription of these phenomena by Bernheim to 'suggestion.' Suggestion has since been investigated from many aspects, down to the work of Coué at the present time, and it has been invoked by Babinski as the essential and finally sufficient explanation of the phenomena of hysteria. The conception involved may be crudely described as the principle that the introduction of an idea, or more properly a conviction, into the mind of an individual will tend to produce certain definite results in that individual. These results may be pathological, as in the production of hysterical symptoms, indifferent, as in the countless examples of suggestion which we see in everyday life, or remedial, as in the practice of suggestion as a therapeutic measure. The conception is clearly a psychological conception, and it has proved its value beyond all question as a weapon in the hands of the practising physician. It is, moreover, a valid conception when examined by the test of its conformity to the rules of scientific method. But it is a conception so vague, and so general in its application to mental processes, that it does not help us far in an understanding of the particular problems presented by disease. Babinski's use of it as a sufficient explanation of hysteria is clearly inadequate, and does not constitute more than a first step in the understanding of that disorder. We want to know why suggestion is so potent in this individual patient, and why certain suggestions are immediately effective in him, while others fail entirely:

We find, indeed, that in this case, as in the conception of dissociation, we have been helped to travel a certain distance, but that the need of a further advance is imperatively felt. The stage in the development of psychopathology to which these conceptions belong is comparable to that existing in the history of astronomy at the time of Kepler. Kepler had shown that the planets move in ellipses round the sun, but he could not explain why they did so. This latter achievement was the work of Newton, with his formulation of the law of gravity. Newton's step was based on the conception that the phenomena observed were the result of certain hypothetical forces, interacting in accordance with certain precisely definable laws. It thus added a dynamic conception as a means of understanding the observed sequence of phenomena. The corresponding step in the construction of a psychological conception capable of taking us beyond the level reached by dissociation and suggestion clearly required a similar advance to a dynamic point of view, and this was, as a matter of fact, the advance 
which was actually attempted at the stage of the history of psychopathology which we are now describing.

This advance was made by Freud, and it constitutes a landmark of the first importance in the development of psychopathology. It marks the essential point of transition from the arid days of the academic psychology, with its meticulous introspective description of mental processes, to the vigorous conceptual and dynamic method of attack which characterises all growing science. Space does not permit of a detailed description of the growth of this dynamic conception, and the general lines of Freud's teaching are now so well known that it is unnecessary to recapitulate them here. It will be profitable, however, to emphasize those broad features which mark the place of Freud's work in the line of historical development which we are considering, and from this point of view the essential principles underlying Freud's conceptions may be sketched as follows. The series of phenomena which constitute conscious life and behaviour are the result of the interaction of a number of psychological 'forces,' acting according to precise psychological ' laws.' $*$ Two or more forces may work harmoniously together, or they may conflict with one another. In the latter case an attempt at adjustment occurs, and certain of these attempted adjustments are of such a kind that morbid phenomena are produced, these morbid phenomena constituting the symptoms observed in certain forms of disorder.

Freud has built upon these basic principles a very elaborate structure, and in it are incorporated many further concepts, amongst which two may be selected for special mention. These are the conception of the unconscious, and the sex theories. Both have been subjected to vigorous attack, partly on grounds which are inadequate and misleading, and it is necessary to deal with these inadequate criticisms before passing on to the problem which is our immediate concern here, the conformity of Freud's teachings to the canons of scientific method.

The conception of the unconscious, formulated by Freud in order to explain the facts of consciousness and behaviour, has been attacked on the ground that it is philosophically untenable and intrinsically absurd. It has been held that mental phenomena must be either conscious or non-existent, and that the notion of unconscious mental processes, therefore, involves an inherent contradiction. This objection rests upon a confusion between phenomena and concepts, and a misapprehension of the function of a scientific concept. The conception of the unconscious has been formulated to explain the observed phenomena, and its validity is no more dependent on its existence as a phenomenal fact than the validity of a weightless, frictionless æther as

* A parallel, and in many essential respects identical, dynamic principle has been reached by other psychologists, in particular by McDougall in his Introduction to Social Psychology. 
a weapon of scientific explanation is dependent upon its phenomenal existence. In both cases the validity of the concept is measured by its utility in resuming, explaining, and enabling us to control the observed phenomena.

Freud's sex theories have been attacked, sometimes explicitly, but more often implicitly, on ethical grounds. Objections of this kind have, of course, no place or relevancy in positive science, and only need to be mentioned in order that they may be at once dismissed.

Freud claims that his doctrines have been built up entirely on an empirical basis, by the observation of the facts of consciousness and behaviour, and the legitimate formulation of concepts to explain those observed facts. There seems good reason to accept, moreover, the frequently made statement that most observers who have investigated these facts by Freud's method have arrived at similar results and have confirmed Freud's teaching. It would seem, also, that Freud's concepts are constructed in a form which is unimpeachable according to the canons of the method of science, and that, if they are based upon observed facts, they satisfy all the requirements of those canons. It is, however, precisely the relation of psychoanalytic doctrine to the observed facts which requires careful investigation and consideration, and there is some reason to question whether the claim that the doctrines are directly based on facts of observation is legitimate. It is true that the doctrines are based on 'facts,' but these facts are not directly observed; they are reached by the employment of a peculiar method, the method of psychoanalysis. This method intervenes, as it were, between the actual facts of observation and the prepared facts upon which the concepts ore based, and it is of such a character that the possibility of distortion cannot with certainty be excluded. The preconceptions of the analyst and of the patient, the deductions made by either or both from the material which rises into consciousness, the stage at which a series of associations is taken to have reached a significant point, all these may be influenced by disturbing factors, and, unfortunately, the influences at work are, at any rate so far as our present knowledge goes, of an incalculable character. It is at least clear that the 'facts' of observation, upon which the Freudian conceptions are based, are of a very different type to those to which we are accustomed in other branches of science. An essential rule of scientific method is that in the construction of concepts and theories a frequent appeal to experience or experiment must be possible, and when made, should yield results consonant with the concept or theory in question. In other words, our course in the regions of conceptual thinking, where it is possible to wander unconstrainedly in almost every direction, must be constantly guided and checked by stepping frequently on to the solid ground of phenomenal experience. We have seen that, in the 
evaluation of Freudian psychology, this appeal is not available in the sense in which it is available in other branches of science. There is an appeal to experience, but this experience is a specially 'prepared' experience.

It is necessary to point out, however, that the defect which has been described is not peculiar to the Freudian methods, but is to some extent inherent in all psychological research. It constitutes, indeed, as Drever has shown, the essential weakness of all psychological method. In psychology the only objective facts are behaviour facts, and in order to deal with them by the psychological method, we require to go behind these facts to the subjective experience underlying them, and thereby to find a new series of facts on which the concepts are ultimately to be constructed. For this reason psychology seems doomed always to occupy an invidious position in the scientific hierarchy, and, hence, explanation of a particular series of phenomena by the concepts of another branch of science is always likely to be accepted in preference to a psychological explanation if both are available.* Nevertheless, the defect under consideration is more glaringly apparent in the Freudian theories than in other instances of psychological method, in that the process of ' going behind' the facts to establish a second series of facts is more extensive and complicated, and takes one further and further from an appeal to phenomenal experience as we are led into the depths of the ' unconscious.' In such relatively simple conceptions as Janet's 'dissociation,' on the other hand, the amount of inferential deduction beyond objectively ascertainable facts is very slight, an appeal to phenomenal experience can be made at almost every step, and the objections on the score of scientific method are, therefore, correspondingly small.

Confirmation of the criticism just put forward is furnished by comparing the widely divergent conceptions reached by different investigators in the analytic field, those, for example, put forward by Freud, Jung and Rivers. In all these different schools of thought the weapons of research are forged of much the same metal, and in not very dissimilar patterns, and yet the results obtained by their use are extraordinarily divergent. Moreover, in face of this divergence, we can make no confident decision between the conflicting claims, because the test of appeal to phenomenal experience, the test by which a similar situation in other branches of science is generally speedily resolved, cannot be adequately and satisfactorily applied.

An attempt may now be made to summarize the position reached by our review of the development of psychopathology. Psychology has clearly established its right to deal with the phenomena of human (hap. I.

* J. Drever, Instinct in Man, Cambridge University Press, 2nd edition, 1921, 
behaviour, and to formulate psychological concepts which will serve to explain those phenomena, provided that they are constructed according to the rule of scientific method. It has to be recognized that psychology is at a disadvantage in that its method is of a character which presents inherent difficulties to the complete satisfaction of those rules, and this disadvantage is equally apparent in the section of psychology constituted by psychopathology. Nevertheless, many of the simpler conceptions of psychopathology, such as dissociation, fail to satisfy the canons of science by so small a margin that it can safely be neglected. In other conceptions, however, particularly those of the analytic schools, the margin is so large that the doctrines of these schools cannot be said to have yet attained the standard which science demands. Yet the islands of rock which dot the sea of analytic speculation are so fertile, and so suggestive of further solid ground extending far around them, that we cannot but feel that ultimately much of that sea will one day be turned into cultivated ground, and that the weapons of analytic research will be shown to be worthy of admission into the accredited armoury of science. The opinion may be ventured that the real need of the moment is the careful examination, testing and perfecting of those weapons, rather than the fashioning of further structures by their aid. 